\title{
Dépistage du dopage aux hormones peptidiques par méthode indirecte : exemple de l'erythropoietine
}

\section{Indirect detection of peptide hormone abuse in doping control : case of erythropoietin}

\begin{abstract}
Michel AUDRAN*(1), Raynald GAREAU(2), Marie-Thérèse SICART ${ }^{(1)}$, Claire CHENARD ${ }^{(2)}$, Bénédicte MARION(1), Guillaume BOUGARD ${ }^{(1)}$, Françoise BRESSOLLE ${ }^{(3)}$
\end{abstract}

(1) Laboratoire de biophysique, Faculté de Pharmacie de Montpellier (2) Département de Chimie Biologie, Université du Québec, Trois Rivières

(3) Laboratoire de Pharmacocinétique, Faculté de Pharmacie de Montpellier

*Auteur à qui adresser la correspondance : Prof. Michel AUDRAN, Laboratoire de Biophysique, Faculté de Pharmacie, 15, avenue Charles Flahaut - 34060 MONTPELLIER Cedex 2 Tél : (33) 467548098 - Fax : (33) 467045323 e-mail : audran@pharma.univ-montpl.fr 


\section{$R E ́ S U M E ́$}

L'érythropiétine humaine recombinante ( $r H u E p o$ ), est utilisée par les athlètes, afin d'augmenter le transport d'oxygène, le pouvoir aérobie et de ce fait, la performance. $L$ 'analogie presque parfaite entre l'érythropoiétine (EPO) physiologique et la rHuEpo et sa faible élimination rénale rendent très difficile la mise au point d'un test de dépistage urinaire fiable. D'autre part sa courte demi-vie et la manifestation tardive de ses effets rendent illusoire son dépistage le jour de la compétition. Nous avons cherché à développer une méthode indirecte de dépistage basée sur la mise en évidence de modifications physiologiques apparaissant lors de l'utilisation de la rHuEpo. Deux protocoles ont été réalisés, le premier en 1995 sur 30 sportifs (20 recevant 5 doses de $200 \mathrm{UI} / \mathrm{kg}$ de rHuEpo par voie sous-cutanée à $48 \mathrm{~h}$ d'intervalle, les 10 autres un placebo); le second en 1997 sur 10 sportifs recevant $50 \mathrm{UI} / \mathrm{kg}$ de rHuEpo et $200 \mathrm{mg}$ de fer par jour durant une période de 26 jours. Outre les paramètres hématologiques nous avons suivi l'évolution du récepteur soluble à la transferrine (sTfR) et de la ferritine (fr) dans le plasma, et des produits de dégradation de la fibrine (TDP) dans les urines. La première étude a permis de mettre en évidence l'intérêt du dosage du sTfR. Les résultats du deuxième protocole confirment l'intérêt de la mesure des sTfR pouvant éventuellement être corrigée par les protéines sériques afin de tenir compte d'une possible hémoconcentration. Cette méthode simple et rapide pourrait constituer une première étape dans le contrôle antidopage lors des compétitions d'autant plus qu'elle permettra également de dépister un dopage par des peptides mimétiques de l'EPO, molécules qui devraient apparấtre sur le marché à partir de l'an 2000.

\section{MOTS-CLÉS}

Erythropoiétine, Dopage, Pharmacocinétique/pharmacodynamie.

\section{Introduction}

Les substances utilisécs par les sportifs pour augmenter leurs performances, peuvent être classées en deux groupes :

les composés exogènes, c'est à dire non présents naturellement dans l'organisme et dont les chefs de file sont les stéroïdes anabolisants et les amphétamines. Leur dépistage bien que tributaire de la limite de détection de la méthode employée, est relativement aisé à condition toutefois de les chercher dans le bon milieu biologique : il est par exemple illusoire de penser retrouver des perfluorocarbures dans les urines.

les composés endogènes tels que la testostérone, l'érythropoïétine ou l'hormone de croissance. Pour ces composés, le domaine des "valeurs biologiques normales" est relativement large de sorte que l'on peut, par apport exogène de ces substances, se doper tout en ayant un taux sanguin ou urinaire "normal" pour la substance en question.

L'érythropoïétine ou EPO, appartient à ce deuxième groupe. C'est un facteur de croissance impliqué dans l'érythro-

\section{SUMMARY}

Recombinant human erythropoitin (RhuEpo) is used by some endurance athletes to increase oxygen transport and aerobic power in an attempt to improve endurance capacity and recovery during competition. The close to perfect homology between endogenous and recombinant Epo, its short plasma half-life and the late clinical manifestation of its effect mean that no reliable analytical technique is yet available to detect its use by athletes. Consequently, we tried to develop an indirect approach to detect rHuEpo misuse. Two protocols have been developed; the first one in 1995 including 30 healthy male athletes $(20$ receiving $200 \mathrm{IU} / \mathrm{kg}$ of rHuEpo subcutaneously every $48 \mathrm{~h}$ and 10 receiving placebo); the other one in 1997 included 9 athletes receiving daily subcutaneous injection of rHuEpo (50 IU.kg) and an oral dose of $200 \mathrm{mg}$ of iron sulphate. Serum erythropoietin concentrations, and haematological and biochemical (ferritin, soluble transferrin receptor (sTfR) and serum proteins) parameters, likewise total degradation products (TDP) in urine, were evaluated during and after treatment. An increased haematocrit with concomitant changes in sTfR and STfR/protein values seems to provide a valuable first step diagnostic tool to allow the identification of rHuEpo abusers.

\section{KEY-WORDS}

Erythropoietin, Doping, Pharmacokinetic/pharmacodynamic.

poïèse.(1-2) Elle stimule la multiplication et la maturation des globules rouges à partir des cellules souches totipotentes de la moelle osseuse. Elle favorise également la synthèse de l'hémoglobine. Elle est, chez l'homme, essentiellement produite par les reins et, à un degré moindre, par le foie. Sa synthèse est induite principalement par l'hypoxie tissulaire. Son taux plasmatique est faible, environ 10 pico$\mathrm{mol} / \mathrm{L}$. Ce taux est généralement exprimé en unités internationales et le domaine des valeurs dites normales s'étend de 2 à 24 UI/L (ou 6 à 32 UI/L selon le kit de dosage utilisé), mais $95 \%$ des sujets se situent entre 6 et $10 \mathrm{UL} / \mathrm{L}$. Glycoprotéine acide de $34 \mathrm{kDa}$., elle est constituée d'une chaîne unique de 165 acides aminés et possède 4 sites de glycosilation, les sucres constituant $40 \%$ de sa masse, et 2 ponts disulfures indispensables à son activité. Elle présente une hétérogénéité moléculaire due à la présence de chaines bi, tri et tetra-anténées. Il existe donc différentes glycoformes. Elle présente également une hétérogénéité dans sa charge : il circulerait 20 à 30 EPO de charge différentes dans le sang.

Au début extraite de l'urine humaine, l'EPO utilisée en thérapeutique est depuis 1987 produite par génie géné- 
tique sous le nom d'érythropoïetine recombinante humaine ou rHuEpo qui est soit l'EPO $\alpha$ commercialisée en France par CILAG (France) sous le nom de EPREX ${ }^{\oplus}$, soit l'EPOB commercialisée par ROCHE-BOERINGERMANNHEIM (Suisse) sous l'appellation RECORMON`. Ces érythropoiétines présentent, comme l'EPO physiologique, une certaine hétérogénéité. La rHuEpo est disponible en France seulement dans les pharmacies hospitalières, ce qui n'est pas le cas dans certains pays limitrophes.

EPO physiologique et rHuEpo sont structurellement très proches : elles ont la même chaîne peptidique et ne diffèrent que par quelques éléments de leur partie glucidique.

Utilisée principalement dans le traitement de l'anémie de l'insuffisant rénal chronique, elle semble avoir fait son apparition dans le milieu sportif en 1988 lors des jeux olympiques d'hiver à Calgary au Canada. L'effet recherché est l'augmentation de la capacité de transport de l'oxygène. Ce dopage ne présente de l'intérêt que dans les sports d'endurance au cours desquels l'énergie est fournie par la voie aérobie c'est à dire par oxydation de l'acide pyruvique et des acides gras. Son utilisation n'est pas sans danger et bien que ses effets à long terme soient encore inconnus, on sait que son utilisation peut conduire à une augmentation de la tension artérielle et à la formation de caillots sanguins pouvant conduire à des accidents pulmonaires, cardiaques ou cérébraux gravissimes.

Le large domaine des valeurs normales de 1'EPO rend impossible la fixation d'un seuil au dessus duquel on pourrait accuser le sportif de dopage. La faible élimination rénale de l'EPO et l'analogie presque parfaite entre la substance physiologique et celle produite par génie génétique rendent très difficile la mise au point d'un test de dépistage urinaire fiable. $D$ 'autre part, sa courte demi-vie et la manifestation tardive de ses effets qui subsistent alors que la molécule incriminée a disparue de l'organisme font que son dépistage le jour de la compétition est peu probable.

Le dopage à l'EPO s'effectue sous forme de cures consistant en injections de 3 "doses" par semaine durant un mois à un mois et demi pour élever l'hématocrite à la valeur souhaitée, puis administration de doses plus faibles, une ou deux fois par semaine pour maintenir cette valeur de l'hématocrite.

Concernant la lutte contre l'utilisation illicite de l'EPO dans le sport, deux approches sont possibles. La première peut être qualifiée de "médecine du travail" et consiste à fixer une valeur limite de l'hématocrite $(50 \%)$ ou de l'hémoglobine $(18.5 \mathrm{~g} / \mathrm{dl})$ à ne pas dépasser pour pouvoir participer à une compétition, et éventuellement à une obligation de se soumettre à des contrôles médicaux réguliers durant l'année. L'autre approche est celle du dépistage du dopage et peut être envisagée de deux façons :

- soit on sépare l'hormone produite par génie génétique l'hormone physiologique, ce type de dépistage semblant plus approprié aux contrôles pendant la cure donc aux contrôles inopinés, hors compétition et vraisemblablement quelques jours après arrêt du traitement,

- soit on cherche un ou des "marqueurs biologiques" dont les valeurs anormalement élevées ou anormalement basses constitueront une preuve indirecte de la prise de rHuEpo ; ce mode de dépistage présentant de l'intérêt aussi bien en cours de traitement qu'après le traitement pourvu que ces marqueurs ne retournent pas rapidement à leur taux normal.

Cette deuxième approche qui caractérise une accélération de l'érythropoïèse aurait l'avantage de pouvoir déceler la prise d'autres composés actifs sur la production des globules rouges tel l'interleukine 3 , le GM-CFS ou plus tard, les peptides mimétiques de l'EPO ; c'est cette approche que nous avons étudié.

Une étude bibliographique avait attiré notre attention sur deux paramètres pouvant constituer des "marqueurs biologiques" de la prise d'EPO, l'un au niveau sanguin : le récepteur soluble à la transferrine ou $\operatorname{sTfR}(3,4)$ l'autre au niveau urinaire : les produits de dégradation de la fibrine ou $\operatorname{TDP}(5,6)$.

\section{Matériel et méthodes}

Deux protocoles ont été réalisés, l'un en 1995 l'autre en 1997, après accord du comité consultatif pour la protection des personnes dans la recherche biomédicale.

Le premier a été effectué sur 30 sportifs, étudiants d'une UFR STAPS, 20 d'entre eux recevant 5 doses de 200 UI $/ \mathrm{kg}$ de rHuEpo (EPREX $\left.{ }^{\circledast}\right)$ par voie sous cutanée à 48 heures d'intervalle, les 10 autres recevant un placebo. Outre les paramètres hématologiques classiques - hématocrite, hémoglobine, réticulocytes, globules rouges- nous avons suivi les variations des concentrations plasmatiques de l'EPO, du récepteur soluble à la transferrine (sTfR), de la ferritine ( $\mathrm{fr}$ ) et au niveau urinaire celle des TDP. L'EPO a été déterminée par dosage radioimmunologique (125 IEPO Coatria ${ }^{\circledR} k i t$, Biomérieux, Lyon, France). Le sTfR, la ferritine et les TDP ont été déterminés par méthode ELISA (respectivement sTfR Ramco Inc., Houston, Texas et Fibrinostika TDP, laboratoire Organon Technica).

Une approche pharmacocinétique/pharmacodynamique de population a permis de modéliser simultanément les variations des concentrations plasmatiques de l'EPO et celles du sTfR de la fr et du rapport sTfR/fr.(7) Une étude préliminaire avait montré que la cinétique de l'EPO, après injection d'une dose unique sous cutanée de $50 \mathrm{UI} / \mathrm{kg}$, était compatible avec un modèle ouvert à 1 compartiment et une vitesse d'entrée d'ordre 0 (8). C'est ce modèle qui a été utilisé pour déterminer les courbes concentration/temps. L'effet a été modélisé comme un compartiment additionnel relié au compartiment central par une cinétique du premier ordre. 
Un deuxième protocole a été réalisé sur 9 sportifs de très bon niveau régional, effectuant entre 20 et 25 heures d'entraînement par semaine. Ces sportifs ont reçu des doses journalières de $50 \mathrm{UI} / \mathrm{kg}$ de rHuEpo et $200 \mathrm{mg}$ de fer (Ferrograd $\mathrm{C} 500^{\circledR}$ ) durant une période de 26 jours ou jusqu'à ce que leur hématocrite atteigne la valeur de $50 \%$ ce qui s'est produit pour 3 d'entre eux. Les paramètres mesurés étaient les mêmes que ceux du premier protocole auxquels a été ajoutée la détermination des protéines sériques.

\section{Résultats}

\section{Protocole 95 :}

Les paramètres hématologiques des sujets recevant l'EPO, exprimés en \% de variation par rapport au groupe témoin sont donnés dans le tableau I.

Tableau I : Variations en \% des paramètres hématologiques des sujets traités à la rHuEpo par rapport au groupe témoin.

\begin{tabular}{|lccccccccc|}
\hline JOURS & $\mathbf{J 0}$ & $\mathbf{J 2}$ & $\mathbf{J 4}$ & $\mathbf{J 7}$ & $\mathbf{J 1 0}$ & $\mathbf{J 1 4}$ & $\mathbf{J 1 7}$ & $\mathbf{J 2 1}$ & $\mathbf{J} 24$ \\
\hline Réticulocytes & 0 & - & 174 & 293 & 263 & 125 & 74 & -15 & -45 \\
Hémoglobine & 0 & $\mathbf{1 , 3}$ & 2,7 & 3,3 & 4,7 & 6,0 & 6,3 & 5,3 & 6,0 \\
Hématocrite & 0 & 2,1 & 2,6 & 4,0 & 3,5 & 5,1 & 6,3 & 5,5 & 6,3 \\
\hline
\end{tabular}

$\mathrm{J} 0, \mathrm{~J} 2, \mathrm{~J} 4, \mathrm{~J} 7$ et J10 sont les jours d'injection de rHuEpo.

Chez les sujets traités, la valeur moyenne de la demi-vie d'élimination de l'EPO est de 42 heures avec une grande variabilité interindividuelle. La concentration en EPO revient à sa valeur de base 7 jours après arrêt du traitement.

Dès le quatrième jour on observe une augmentation du sTfR dont la valeur devient significativement différente de celle des sujets témoins (fig.1). Cette valeur devient 2 fois supérieure à celle du groupe témoin dès le septième jour du traitement et atteint même 3 fois la valeur normale le septième jour suivant la dernière injection avant de revenir lentement à sa valeur normale : 14 jours après arrêt du traitement, la valeur moyenne du sTfR est deux fois plus élevée chez les sujets traités que dans le groupe témoin.

Afin de tenir compte d'une éventuelle hémoconcentration ou d'une hémodilution il nous a semblé intéressant de corriger la valeur du sTfR par la ferritine. L'écart entre le groupe traité et le groupe témoin est alors amplifié, la valeur du rapport sTfR/fr devenant 6 à 14 fois plus élevé dans le premier groupe fig (2). Ceci peut s'expliquer par le fait que l'accélération de l'érythropoï̀se abaisse les réserves en fer. On notera cependant que le rapport sTfR/fr revient plus rapidement à sa valeur normale que le sTfR.

$\mathrm{Au}$ niveau urinaire l'analyse des TDP n'a fourni aucun résultat probant.

Il apparait donc que le sTfR semble être le paramètre indirect le mieux approprié pour détecter la prise de rHuEpo et ce d'autant plus que la diminution de la ferritine peut être minimisée, voire annulée, par une supplémentation convenable en fer.

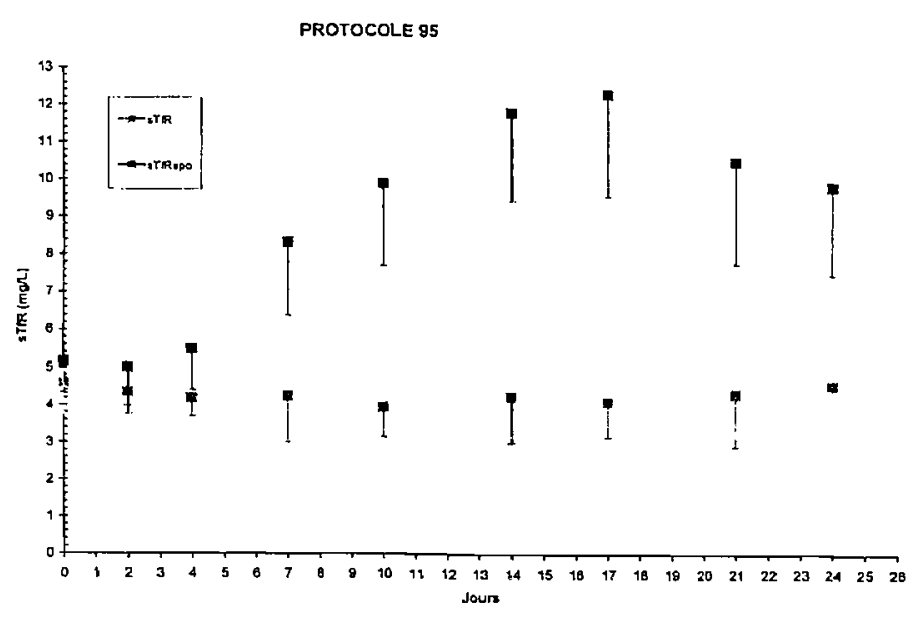

Figure 1 : Comparaison des concentrations sériques en sTfR au cours du temps chez le groupe de sujets traités à la rHuEpo (무) et chez le groupe témoin( $\bullet$ ).

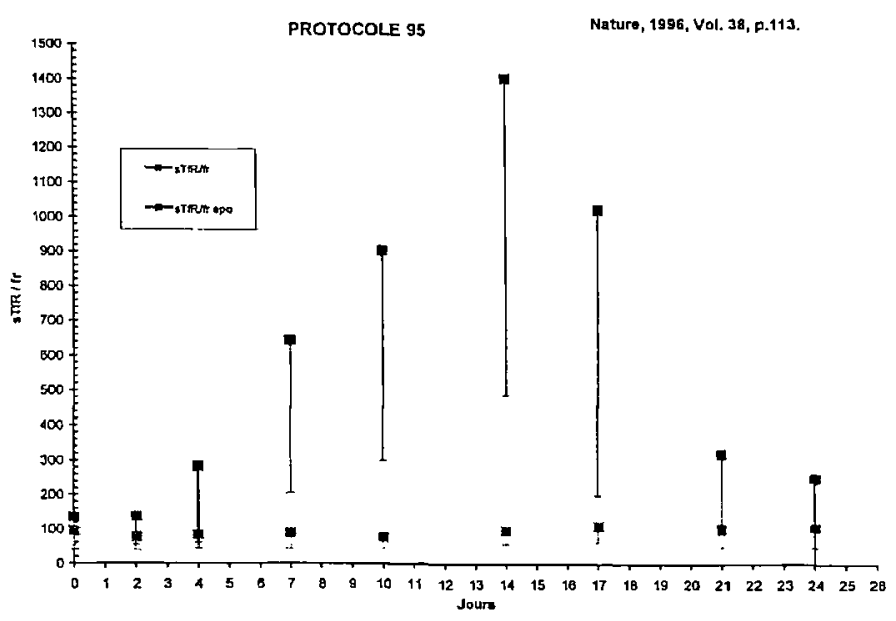

Figure 2 : Comparaison des rapports sTfR chez le groupe de sujets traités par la rHuEpo (G) et le groupe témoin( $\bullet)$.

\section{Protocole 97}

Les valeurs des paramètres hématologiques déterminés lors du second protocole sont présentés sur les figures ( 3 , $4,5,6,7,8)$. On notera une augmentation de l'hématocrite mais celle ci ne devient significative qu'à partir du $24^{\text {cme }}$ jour de traitement. Comparée au premier jour de l'étude, cette augmentation est, en fin de traitement, de $11 \%$; elle se maintient une semaine environ puis la valeur de l'hématocrite redescend vers sa valeur normale. La variation du taux d'hémoglobine est tout à fait comparable à celle de l'hématocrite : la valeur maximale, atteinte en fin de traitement est de $9 \%$ supérieure à celle du départ. Elle se maintient une semaine environ avant de revenir à sa valeur d'origine. Le nombre des réticulocytes augmente très rapidement et la différence entre les valeurs mesurées le $1^{\text {er }}$ et le $10^{\text {emc }}$ jour est hautement significative. Cette augmentation reste significative pendant la durée du traitement et les 3 jours suivants la dernière injection avant de redescendre et de devenir inférieure à la valeur normale 13 jours environ après arrêt du traitement. Cette valeur, anormalement basse, subsiste une dizaine de jours environ. On notera cependant que pour tous ces paramètres 


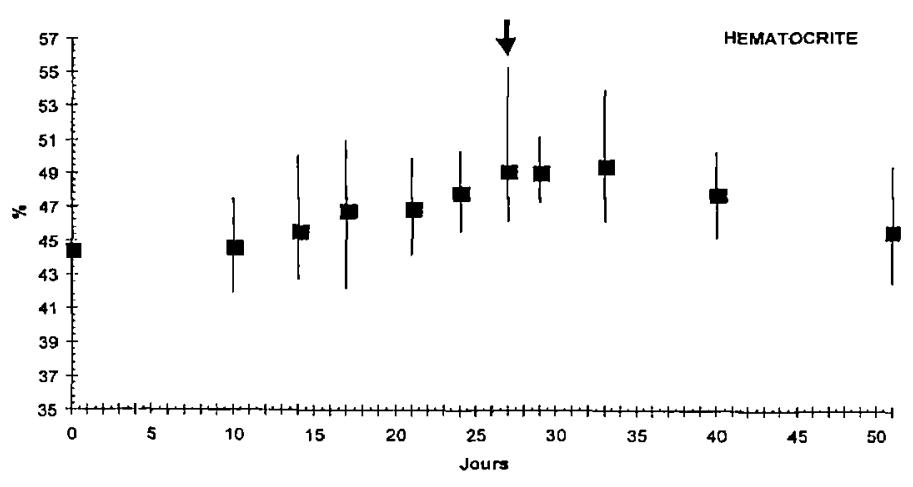

Figure 3 : Variations du taux d'hématocrite pendant et après injection de rHuEpo. Les traits verticaux représentent le domaine des valeurs mesurées (et non un écart-type). Le cadre délimite les valeurs maximales et minimales des sujets avant prise de rHuEpo. La flèche représente le jour d'arrêt du traitement.

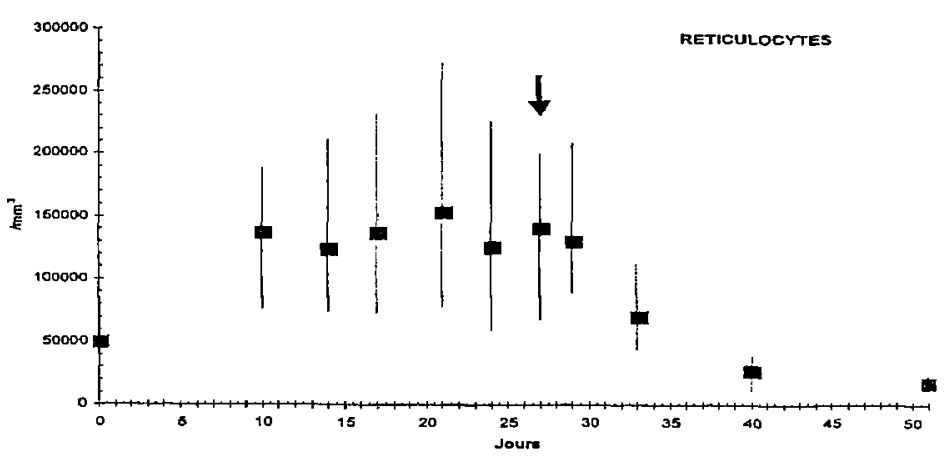

Figure 5 : Variations du taux de réticulocytes avant et après administration de rHuEpo. Les traits verticaux représentent le domaine des valeurs mesurées (et non un écart-type). Le cadre délimite les valeurs maximales et minimales des sujets avant prise de rHuEpo. La flèche représente le jour d'arrêt du traitement.

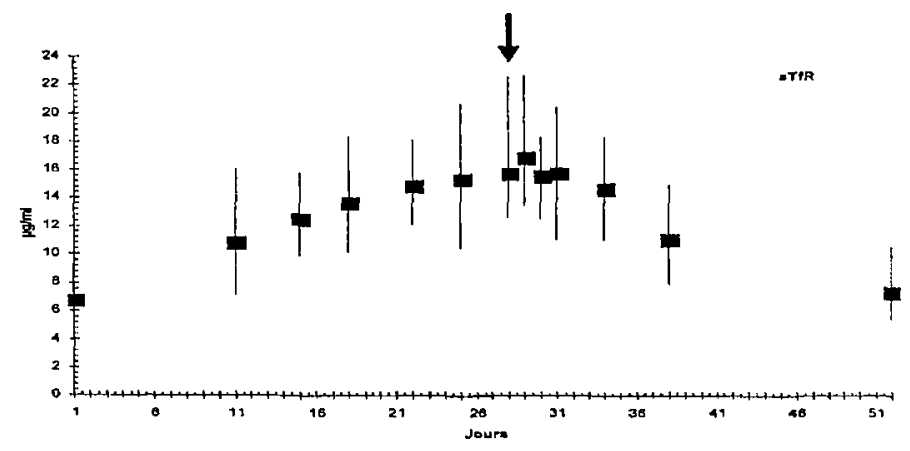

Figure 7 : Variations de la concentration sérique du sTfR pendant et après administration de rHuEpo. Les traits verticaux représentent le domaine des valeurs mesurées (et non un écart-type). Le cadre délimite les valeurs maximales et minimales des sujets avant prise de rHuEpo. La flèche représente le jour d'arrêt du traitement.

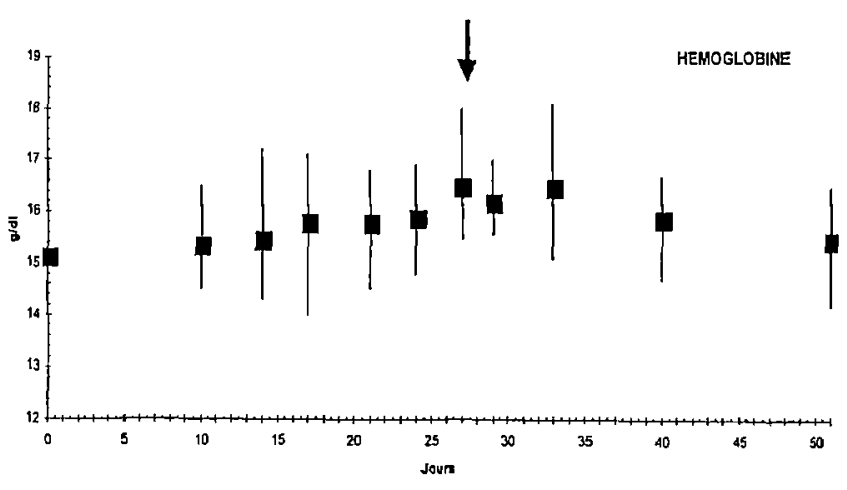

Figure 4: Variations du taux d'hémoglobine pendant et après injection de rHuEpo. Les traits verticaux représentent le domaine des valeurs mesurées (et non un écart-type). Le cadre délimite les valeurs maximales et minimales des sujets avant prise de rHUEpo. La flèche représente le jour d'arrêt du traitement.

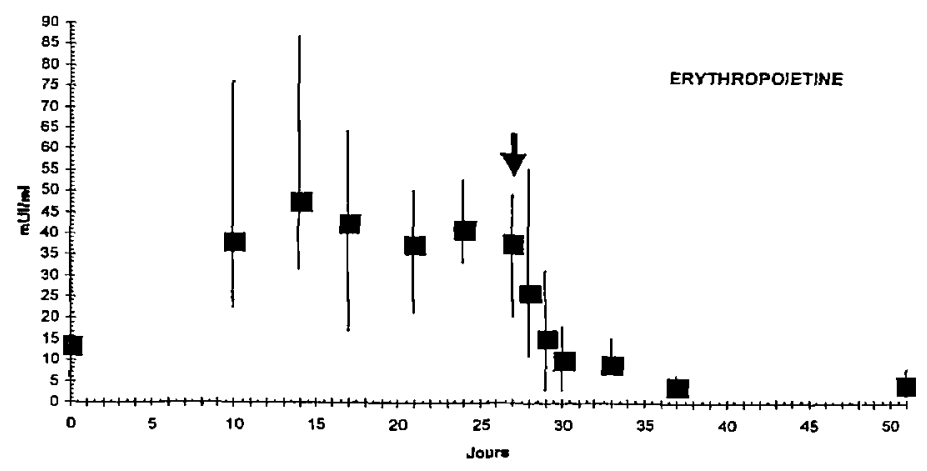

Figure 6 : Variations de la concentration sérique de l'EPO pendant et après administration de rHuEpo. Les traits verticaux représentent le domaine des valeurs mesurées (et non un écart-type). Le cadre délimite les valeurs maximales et minimales des sujets avant prise de rHuEpo. La flèche représente le jour d'arrêt du traitement.

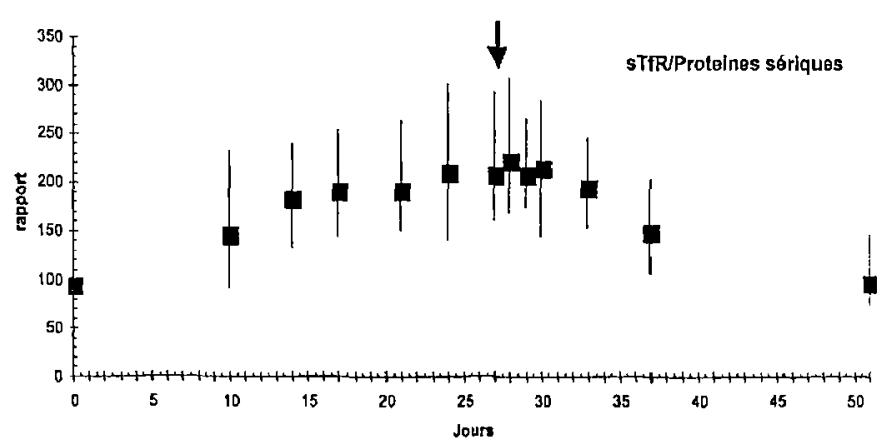

Figure 8 : Variations du rapport sTfR/protéines pendant et après administration de rHuEpo. Les traits verticaux représentent le domaine des valeurs mesurées (et non un écarttype). Le cadre délimite les valeurs maximales et minimales des sujets avant prise de rHuEpo. La flèche représente le jour d'arrêt du traitement. 
certains sujets demeurent dans les domaines de valeurs normales à l'exception de la valeur anormalement basse des réticulocytes qui dès le $13^{\text {enc }}$ jour après le traitement affecte l'ensemble des sujets.

Le profil de l'EPO est semblable à celui des réticulo-

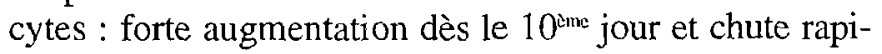
de à l'arrêt du traitement avec des valeurs anormalement basses pour l'ensemble des sujets à partir du $10^{\text {enc }}$ jour d'arrêt. Cet état, semblable à un phénomène de rétro inhibition, se maintenant deux semaines environ.

sTfR et sTfR/protéines montrent des cinétiques comparables : forte augmentation observée dès le $10^{\text {ime }}$ jour et valeurs maximales atteintes 1 à 2 jours après arrêt du traitement. Du $14^{\text {ame }}$ jour de traitement au $7^{\mathrm{z} m \mathrm{e}}$ jour après arrêt des injections d'EPO, tous les sujets, sans exception, présentent des valeurs supérieures au domaine des valeurs normales. Le retour à des valeurs normales se fait plus ou moins rapidement selon les sujets, 5 sportifs sur 9 étant encore largement au dessus de celles ci deux semaines après arrêt des administrations d'EPO.

Dans cette étude également, la détermination des TDP urinaires n'a donné aucun résultat intéressant.

\section{Discussion}

Ces études démontrent que le sTfR, éventuellement corrigé par les protéines plasmatiques de façon à tenir compte d'une éventuelle hémoconcentration ou hémodilution, présente un intérêt certain pour diagnostiquer la prise de rHuEpo. Mais la prise d'EPO n'est pas le seul facteur susceptible d'entraîner une augmentation du sTfR ou du rapport sTfR/protéines : ces paramètres augmentent dans certaines pathologies - le sTfR est un bon marqueur de la déficience en fer-mais ces pathologies sont aisées à diagnostiquer et incompatibles avec la compétition sportive.

Nous avons mesuré la valeur du sTfR et celle du rapport sTfR/proteines dans différentes conditions :

- sur 6 sujets au départ et à la fin d'un semi-marathon

- sur 10 sportifs de haut niveau effectuant en stage en altitude à 1800-2000 mètres ( les prélèvements ont été faits au moment de l'arrivée, le cinquième et le huitième jour du stage)

- sur 9 sujets après 5 jours passés à 4300 mètres

- sur 23 autochtones vivant à 3600 mètres

- sur 21 femmes au cours de la première partie et de la deuxième partie de leur cycle menstruel.

Nous avons pu constater que ni l'effort, ni l'altitude, ni le cycle menstruel chez la femme ne modifiaient les valeurs de ces paramètres.

En incluant à ces résultats les 176 déterminations effectuées sur les athlètes participant aux championnats de France de VTT (tous prélevés le matin des compétitions cntre 8 heures et 10 heures) nous avons pu établir statistiquement que une valeur de $10 \mu \mathrm{g} / \mathrm{ml}$ pour le sTfR et 150 pour le rapport sTfR/protéines pouvaient constituer des valeurs seuils au dessus desquelles il y avait lieu de soupçonner un dopage.

\section{Conclusion}

On peut donc conclure, avec un risque d'erreur inférieur à $1 \%$, qu'en présence d'un taux d'hématocrite élevé et de valeurs du sTfR ou du rapport sTfR/protéines supérieures aux valeurs seuils on est en présence d'un cas de dopage.

Cependant ces chiffres sont à considérer avec une certaine réserve : ils sont tributaires des méthodes de détermination des deux paramètres et notamment du sTfR. : les différents kits disponibles pour le dosage de ce dernier ne donnant pas les mêmes valeurs. D'autre part ce seuil calculé pour le sTfR est supérieur à la valeur considérée comme pathologique $(8 \mu \mathrm{g} / \mathrm{ml})$ par le fabricant du kit et peut être exagérément élevée et conduire à un nombre non négligeable de "faux négatifs".

Deux paramètres peuvent sembler insuffisants pour affirmer qu'il y a eu accélération illicite de l'érythropoïèse. Mais la détermination des réticulocytes et celle de l'EPO, les deux étant anormalement élevés durant la prise d'EPO et anormalement bas sur une période allant de dix à vingt jours après arrêt du traitement, peuvent constituer des arguments non négligeables pour affirmer ou infirmer la suspicion de dopage. La certitude pourrait être apportée en effectuant deux ou trois prélèvements inopinés dans la semaine suivant le premier prélèvement, mais une telle procédure semble, pour l'instant, utopique.

\section{Références}

1. Beguin Y., The soluble transferrin receptor : biological aspect and clinical usefulness as quantitative measure of erythropoiesis. Haematologia, 1992, $77: 1-10$.

2. Beguin Y., Clemons GK., Pootrakul P., Fillet G., Quantitativeassessment of erythropoiesis and functional classification of anemia based onmeasurement of serum transferrin receptor and erythropoietin. Blood, 1993, 81, 1067-1076.

3. Gareau R, Brisson GR., Ayotte C., Dubé J., Caron C., Erythropoietin doping in athletes : possible detection though measurement of von Willebrand and D-dimer activity ? Thrombosis and Haemostasis, 1992, $68: 481-482$.

4. Gareau R., Gagnon MG., Ayotte C., Chénard C., Brisson GR., rHuEPO increases urinary excretion of fibrin degradation products in heamodialysed patients. Thrombosis and Haemostasis 1993, 70 : 373-374.

5. Souillard A., Audran M., Bressolle F, Gareau R., Duvallet A., Chanal IL. Pharmacokinetics and pharmacodynamics of recombinant human erythropoietin in athletes. Blood sampling and doping control.Br J Clin Pharmacol., 1996, 42 : 355-364.

6. Bressolle F., Audran M., Gareau R., Baynes RD., Guidicelli C., GomeniR. Population pharmacodynamics for monitoring Epoetin in athletes, Clin Drug Invest., $1997,14: 233-242$. 